\title{
Digital Preoperative Planning for High Tibial Osteotomy using 2D Medical Imaging
}

\author{
Norazimah Awang ${ }^{1 *}$, Faudzi Ahmad ${ }^{2}$ \\ Rosnita A. Rahaman ${ }^{3}$ \\ Computing Department, Universiti Selangor \\ Malaysia
}

\author{
Riza Sulaiman $^{4}$ \\ Institute IR 4.0 \\ Universiti Kebangsaan Malaysia \\ Selangor, Malaysia
}

\author{
Azrulhizam Shapi' ${ }^{5}$ \\ Centre for Artificial Intelligence Technology \\ Universiti Kebangsaan Malaysia \\ Selangor, Malaysia \\ Abdul Halim Abdul Rashid ${ }^{6}$ \\ Orthopaedic Department \\ Universiti Kebangsaan Malaysia Medical Center \\ Selangor, Malaysia
}

\begin{abstract}
The pre-operative planning process for High Tibial Osteotomy (HTO) is vital to correct the deformity of the long bones. The most important process is needed to find the Centre Of Rotation of Angulation (CORA) and display the forecast result based on the value of the correction angles simultaneously. Presently, these practices should be done manually because current software only can define either CORA's point or correction angle at one time. This paper proposed to use computer-aided software to make the fully digitized process of pre-operative planning for HTO to be done. For this purpose, we introduced OsteoAid software. This software enables the user to define the mechanical or anatomical axes and define the CORA's point and the angle at one time. For testing purposes, we compared the reliability of osteotomy's correction angle between this two software (MedWeb and OsteoAid) in preoperative planning open-wedge high tibial osteotomy. This is to ensure that the new software is reliable for the correction. Thirteen digital long leg radiographs with long-standing positions from the frontal axis showing patients with both tibia deformities were examined using intra-class correlation. Those images are accessed from the picture archiving and communication system (PACS). Three medical officers (raters) who were involved in an osteotomy used the same medical image format twice with a twoweek interval. Using the MedWeb software, the mean correction angle score of each rater is at excellent level: 0.989 (intra-rater1), 0.982 (intra-rater2) and 0.972 (intra-rater3). Scores of each rater for OsteoAid are also excellent: $0.949,0.987$ and 0.986 respectively. The inter-rater reliabilities of the correction angle were 0.820 and $0.979(p<0.001)$ respectively for each software. The principal finding of this study was that the new software (OsteoAid) showed excellent reliabilities and good consistency in preoperative planning in finding CORA and correction angle.
\end{abstract}

Keywords-Center of rotation of angulation; CORA; HTO; software; digital; medical image

\section{INTRODUCTION}

The High Tibial Osteotomy (HTO) is one of the trusted methods and an effective medical treatment option for correction in ensuring the success of HTO surgical procedures [1], [2]. HTO is an acceptable and common procedure to treat symptomatic osteoarthritis of the medial compartment of the knee with varus alignment. HTO is considered a very successful surgical procedure with low complication rates. The goal of surgical treatment is to shift the weight-bearing axis from the medial compartment to the lateral compartment [3]. It is becoming increasingly important nowadays in the treatment of cartilage pain or central osteoarthritis with varus deformity [4].

Proper planning, especially the height of the wedge base and osteotomy correction angle, is also a crucial aspect of effective HTO. Usually, preoperative planning of HTO will be done before the real surgery to ensure optimum correction and to avoid malalignment that can lead to under-correction or over-correction, even with navigation systems [5]. Inaccurate preoperative planning will produce inadequate correction during surgery and loss of correction in the postoperative period [6].

Previously, conventional preoperative planning for HTO was done by tracing out the X-ray film on paper. When the technology was replaced from X-ray film to digital, the full digital procedure for preoperative planning should also be produced. Current software is reliable for preoperative planning for HTO in a part of procedure such as finding CORA's point or angle at one time only, it does not visualise the forecast result as needed by surgeon. This motivates researchers to develop a new software that can fulfil all the requirements needed by surgeon.

Computer-assisted planning gives the advantages to the higher accuracy and reliability in many ways such as digital storage, infrastructure and technology [7], digital planning [3], better result [8], real-time feedback [9] and possible to display future results [10]. In optimizing a digital radiographic image in the orthopaedic department, the researcher developed digital preoperative planning for high tibial osteotomy software.

A previous study from Awang et al [11] shows five different specification software namely TraumaCad, MediCad, Sectra, MedWeb, and Photoshop applied to implement preoperative planning for an osteotomy. However, certain software is usually equipped with advanced equipment

\footnotetext{
*Corresponding Author
} 
(computer navigation) and exorbitantly costly and it is difficult for developing countries such as Malaysia to purchase. On the other hand, current software implemented in Universiti Kebangsaan Malaysia Medical Center (UKMMC), as an example, has limited functions and does not provide a forecast image for postoperative.

OsteoAid software was developed to overcome the current limitation that occurred during the planning process, such as, the current software does not provide a centre of rotation of angulation and it does not provide visualisation for correction. The new software enables the user to easily find out an angle for correction and rotate a part of the image and visualise it. This paper aims to determine the CORA and correction angle at one time. It also aims to compare the accuracy of new software, in order to ensure the reliability of the new software in doing preoperative planning for HTO.

Meanwhile, the CORA is the point of intersection between two straight lines at the proximal and distal [12]. According to Kim et al., [3] CORA is determined by the intersection of the transverse dividing line between the proximal and distal tibial anatomical axes and the posterior tibial cortex. Moreover, it allows accurate analysis of lower limb angular defects [13].

CORA is the most important assessment for angular defect correction. The line that crosses the CORA and divides the lateral and medial angles formed by the proximal and distal axes of the bone is called the transverse bisector line (tBL).

The intersection of these straight lines involves mechanical [14], [15] or anatomical axes [16] It is also influenced by the need or regulation of the osteotomy itself i.e. varus gene defect [14], valgus [17] or recurvatum [18]. This selection also depends on the selection of wedges to be used that is whether open wedge, closed, dome and also a combination.

Thus, this study is focusing on the requirements needed to develop the digital planning for HTO. CORA as the main requirement in this study is needed to guide the surgeon to cut the proximal tibia with dedicated correction angle. Then the correction angles founded from the same images using two different software were then tested and compare their ICC to prove that it is also equivalent among MedWeb and OsteoAid. This section also contains the background of the current research. Section II discussed the materials and methods needed in this study. Section III discussed the evaluation followed by the results, discussion and conclusion.

\section{MATERIALS AND METHODS}

This new software was developed based on the user requirements. The main focus of this development is on the defining CORA's location and correction angles' value. Besides, this software enables end user to see a picture based on the previous result of CORA point and the angle of the correction.

A retrospective case series was conducted between 2016 and early 2018, 26 tibia's radiographic images from 13 patients have already undergone osteotomy surgery. The patients were five men and eight women with aged between two to 15 years old. To ensure good quality results, two identical tests will be conducted by the same three evaluators for all two tests, within a two-week interval at the PPUKM Orthopaedic Clinic by medical officers in specialist training in the osteotomy field.

The three medical officers (evaluators) will test the Medweb software and also OsteoAid using medical images (Dicom). All of the images were tested to compare CORA's angle finding from two different software; MedWeb which is the current software used in that hospital and OsteoAid, new proposed software that was developed to overcome MedWeb's deficiency.

\section{A. Preoperative planning}

Since this study focused only on the genu varum and valgus, all radiographic images were from the coronal plane which required a long lower limb. The aim is in preoperative planning for high tibial osteotomy by realigning the deformity leg to the normal weight-bearing line. This study only focuses on comparing results in finding CORA based on two different softwares. Here, CORA was defined based on two intersections of lines produced from proximal and diaphyseal anatomical axis to produced lateral open wedge for high tibial osteotomy. Fig. 1 shows how to produce CORA and the correction angle.

\section{B. Radiographic Evaluation}

There are many records of patients who had undergone osteotomy, but to ensure that the testing can be done fairly and transparently, several things need to be concentrated. Those requirements are long leg radiograph with long-standing position from the frontal axis and using the same Movement Reference Number (MRN) of each patient from picture archiving and communication system (PACS).

\section{Correction Angle}

A correction angle is an angle produces based on the percentage of anatomy or mechanical axis. An example, based on [19], the target mechanical axis were calculated as much as $62.5 \%$ using radiographs in the standing and supine positions based on Fujisawa technique. The author in [17] defines correction angle based on the drawn line from the hinge point to the line connecting the center of the femoral head and 55, 60 , and $65 \%$ weight-bearing line.

In this study, two straight lines of $\mathrm{T}$-shaped and $\mathrm{T}$ downward which are known as T1 and T2's markers were used to produce the angles. T1 as by default is at a zero angle of degrees while T2 is at an angle of 180 degrees. If the user clicks on the right arrow key for any marker, the current value of the marker will be added to five degrees, while if the user clicks on the left arrow key, the current value of the marker will be subtracted to negative five degrees. Those markers are shown in Fig. 1.

Similarly, if the user clicks on the up-arrow key, the current value of the marker will be added to one degree, while if the user clicks on the down arrow key, the current value of the marker will be subtracted to negative one degree. The two markers will then be continuously updated with the value of their respective findings. Fig. 2 shows the programming language used to calculate the angles. 


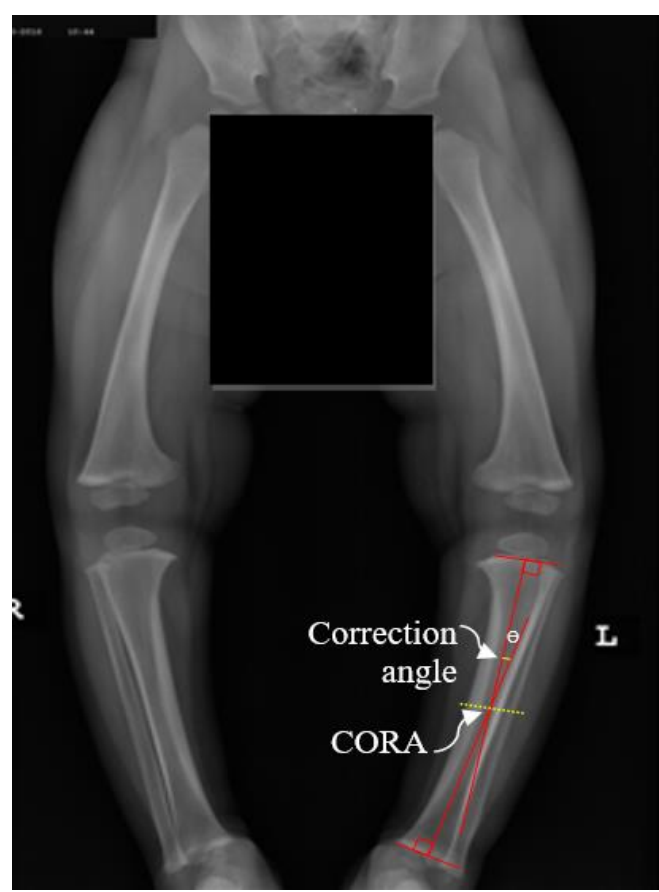

Fig. 1. CORA's Location and Correction Angle.

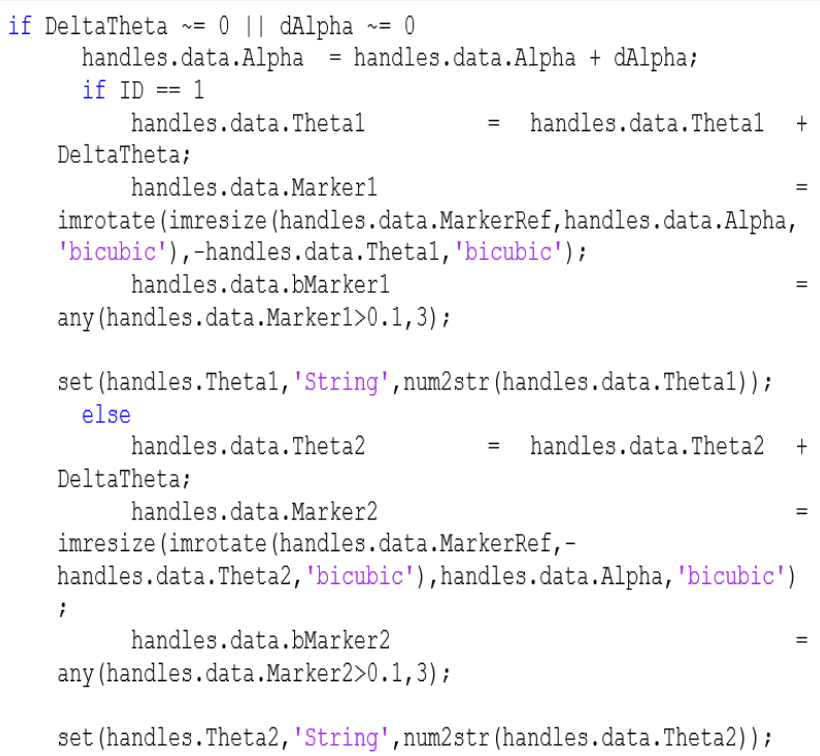

Fig. 2. Algorithm of the Selection and Rotation a Part of Image.

\section{Rotating a Part of Image}

As the correction angle is produced, they want to display the prediction of correction. Based on the CORA correction's angle obtained, the next task is to mark an area of the image of the tibia to be rotated and visualize the expected result of the rotation. This is done to get the satisfaction of the surgeon in obtaining the best picture of point and angle results before the operation. In addition, it is also important to provide an initial overview to patients and family members about the treatment methods that the patient will receive.

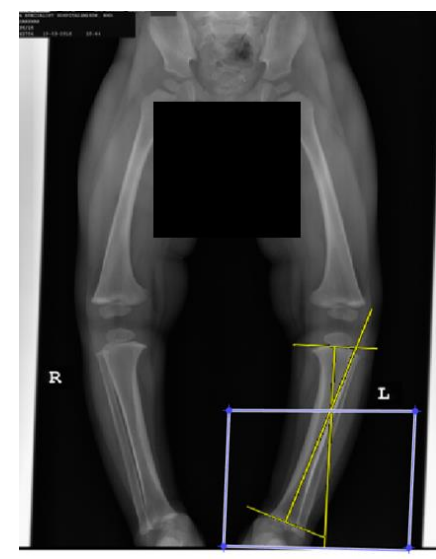

(a)

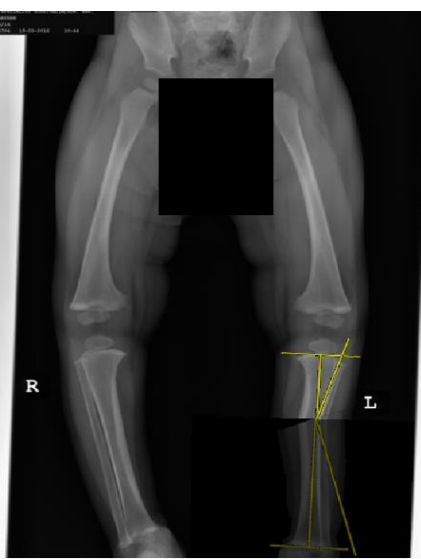

(b)
Fig. 3. (a) Process of Selection a Part of the Area, (b) Result Displayed based on the Correction Angle.

Fig. 3(a) shows an example where a part of the area was selected for cropping and rotation. Here, the user needs to mark four corners of the part of the image to be rotated and it must cross the line at the CORA point. In the last corner, which is the fourth corner, the user has to click the mouse twice to finish the process of selection. Then the result will display as shown in Fig. 3(b).

\section{E. Statistical Analysis}

Normality tests were performed using Kolmogorov Smirnov on all variables before determining parametric statistics. This test is made to determine the compatibility between the two methods is also tested by comparing the mean of the correction angle obtained. Kolmogorov - Smirnov testing was performed to confirm the normal distribution. This test was chosen to test the normality of the data when the data were in small numbers and less than 50.

If the data is normal, then the researcher can use a T-test to run a further test. Else, Wilcoxon signed-rank test can be used. Then, the intra-class correlation was used to test the reliability of the results (ICC). Inter- and intra-rater reliabilities using the IntraClass Correlation Coefficient method were accessed. For statistical analysis, the IBM SPSS software for Windows version 17 was employed to determine if statistically significant similarities were present between the group studied. A p-value of 0.05 or less was considered to be a significant difference.

\section{EvaluATION}

After the development of the system was completed, one simple test will be done to evaluate the accuracy of the system. Testing has been conducted on 13 patients with impairment on both legs who had undergone osteotomy treatment from 2016 to early 2018 at Universiti Kebangsaan Malaysia Medical Center (UKMMC). The respondents comprised of three medical officers who practice osteotomy correction planning and the tests were conducted at UKMMC. They tested on this system themselves in conducting osteotomy preoperative planning procedures and obtained the results based on the given patient. To determine the sample size, an a priori power analysis was performed using the null hypothesis test at alevel 
of .05. It had been hypothesized that the reliability of intra- and inter-rater for the dedicated software was zero.

As the study involves the field of medicine that leads to orthopaedic, the test is based on the guidelines obtained from the International Society of Arthroscopy, Knee Surgery and Orthopaedic Sports Medicine (ISAKOS), in which the results lead to the reliability of new techniques based on existing techniques.

The reliability of this new software will be evaluated. The current software is regarded as a gold standard, and consistency was evaluated for the examination of the accuracy of the new system PACS-OsteoAid method. Coronal alignment and posterior tibia slope were evaluated and excluded to sagittal alignment. The reliability of the measurement was accessed, by examining the intra-rater and inter-rater reliability, using intra-class correlation coefficient (ICC) to determine each component score [20]. Three medical officers, who were involved in osteotomy, measured the correction angle twice with a two-weeks interval.

\section{RESUlts}

A few standards on the development that have been done were listed to be followed before testing could be executed as followed:

1) the software can use the medical image in Dicom format.

2) surgeons are free to define CORA.

3) the software can visualise CORA for correction.

4) software is tested by the medical officer who is involved in osteotomy.

Based on the standard proposed, one evaluation was done to accept the level of accuracy achieved from the usage of OsteoAid. The demographic data based on thirteen patients with both tibia's patients underwent preoperative planning in finding CORA angle are in age 2-15 based on the data available in the PACS is shown in Table I.

Table II shows the two tests (Test1 and Test2) that need to be performed by all evaluators. Result shows that all of the significant value is 0.00 . This indicates that Test 1 and Test 2 are not significantly different from the normal distribution based on statistics. Thus, non-parametric testing such as Wilcoxon Rank-Marked Test will be conducted.

The Wilcoxon Signed-Rank Test was chosen to make a comparison between Test 1 and Test2. If a pair of scores has the same value, then it is considered bound and dropped from the analysis and the sample size will be reduced taking into account the value of $\mathrm{P}<0.05$ is considered significant. For this study, the Wilcoxon Rank-Marked Test was used to compare scores from two tests performed at two different time points.

The finding of MedWeb's for Rater1 and Rater2 from two weeks apart for Rater1 did not show a statistically significant change $(p=.444,0.71)$ compared with alpha value $=0.05$. For Rater3, it shows a statistically significant change $(p=.036)$ compared with alpha value $=0.05$. For the OsteoAid, all raters did not show a statistically significant change $(p=.545, .224$, .932) compared with alpha value $=0.05$.
TABLE I. DEMOGRAPHIC RESULT

\begin{tabular}{|l|l|}
\hline Item & Number \\
\hline Total tibia examined & 26 \\
\hline Total number of patients & 13 \\
\hline Right tibia & 13 \\
\hline Left tibia & 13 \\
\hline Age & $2-15$ \\
\hline Gender (M/F) & $5 / 8$ \\
\hline
\end{tabular}

TABLE II. NORMALITY TEST BY KOLMOGOROV-SMIRNOV

\begin{tabular}{|l|l|l|l|}
\hline & \multicolumn{3}{|l|}{ Kolmogorov-Smirnov } \\
\hline & Statistic & df & Sig \\
\hline Test1 & $\mathbf{. 1 1 1}$ & $\mathbf{2 3 4}$ & $\mathbf{. 0 0 0}$ \\
\hline Test2 & $\mathbf{. 0 9 0}$ & $\mathbf{2 3 4}$ & $\mathbf{. 0 0 0}$ \\
\hline
\end{tabular}

As shown in Table III, the finding of MedWeb's for Rater1 and Rater2 is there is no difference between Testing1 and Testing2 whereas for Rater3, there is a difference between Testing1 and Testing2. For the OsteoAid, the findings for each rater are there was no difference between Test 1 and Test2. The factor that contributes to the difference between Testing 1 and Testing 2 for Rater3 was due to the factor of variability between users where this factor is out of control. Thus, the number of evaluators of three people has helped to reduce the biased results.

TABLE III. WILCOXON SIGNED-RANK TEST

\begin{tabular}{|l|l|l|l|l|}
\hline \multirow{2}{*}{ Rater } & \multicolumn{2}{|l|}{ MedWeb } & OsteoAid \\
\cline { 2 - 5 } & z value & $\boldsymbol{p}$ Value & z value & $\boldsymbol{p}$ Value \\
\hline Rater1 & -.765 & .444 & -.605 & .545 \\
\hline Rater2 & -1.806 & .071 & -1.217 & .224 \\
\hline Rater3 & -2.102 & .036 & -.086 & .932 \\
\hline
\end{tabular}

Table IV shows the intra-rater result for each rater towards each software. For intra-raters, three raters would test two different software which is (a) MedWeb and (b) OsteoAid. Every rater had done the testing twice every fortnight. Meanwhile, the inter-rater testing was done by all three raters towards the two software. Through the statistics software package SPSS version 21 (SPSS Inc.), an estimation of ICC and Confidence interval (CI) of 95\%, absolute-agreement and two-way mixed-method model were chosen.

Each rater scored excellent level: 0.989 (0.975-0.995), 0.982 (0.961-0.992) and 0.972 (0.938-0.987) respectively for MedWeb. As for OsteoAid, the scores were also excellent: 0.949 (0.882-.0978), 0.987 (0.970-0.994) and 0.986 (0.9690.994) respectively. The finding from the usage of each software to get the CORA angles of the preoperative planning for osteotomy by all three raters is excellent.

The statistics for the inter-rater reliability between MedWeb and OsteoAid showed that the ICC of the latter is higher compared to the former. The ICC for OsteoAid is 0.979 while the ICC for MedWeb is at 0.820. Therefore, both figures can be accepted. OsteoAid method is in the range of good and acceptable reliability for preoperative planning of high tibial osteotomy. 
TABLE IV. INTRA-CLASS CORRELATION FOR INTRA-RATERS

\begin{tabular}{|l|l|}
\hline Rater & Correction Angle \\
\hline Intra-rater 1(a) & $0.989(0.975-0.995)$ \\
\hline Intra-rater 2(a) & $0.982(0.961-0.992)$ \\
\hline Intra-rater 3(a) & $0.972(0.938-0.987)$ \\
\hline Intra-rater 1(b) & $0.949(0.882-0.978)$ \\
\hline Intra-rater 2(b) & $0.987(0.970-0.994)$ \\
\hline Intra-rater 3(b) & $0.986(0.969-0.994)$ \\
\hline Inter-rater (a) & $0.820(0.663-0.901)$ \\
\hline Inter-rater (b) & $0.979(0.967-0.987)$ \\
\hline
\end{tabular}

Intra-rater 1 means ICC of rater 1 for first and second measurements. Intra-rater 2 means ICC of rater 2 for first and second measurements. Intra-rater 3 means ICC of rater 3 for first and second measurements. (a) refers to MedWeb, while (b) refer to OsteoAid. Inter-rater (a) means the ICC using MedWeb, between rater 1,2 and 3. Inter-rater (b) means the ICC using OsteoAid between rater 1,2
and 3.

\section{DISCUSSION}

The most important finding of this study was that new software (OsteoAid) had a significant reliability in finding CORA's angle rather than current software (MedWeb). This study successfully compares measurement angles of digital radiographic images among two softwares. In addition, there was excellent intra- and inter-rater correlation.

Planning techniques need to be reliable [21]. This study was done because existing software can only perform one of the procedures at a time. It is difficult for surgeons to define the CORA and propose the correction angle at one time. Fig. 4 shows the CORA that was produced based on the intersection of two lines, and the correction angle is automatically displayed when the user rotates the line based on the desired location.

Other studies have discussed the preliminary planning of high tibial osteotomy [14], [22]. Based on different osteotomy problems, the need for planning also varies. For example, based on the difference of deformity whether genu varum, valgus or recurvatum, selection of frontal and sagittal planes, selection of mechanical or anatomical axis [22] in planning, open or close wedge, as well as CORA cuts are also different is based on the needs and determined by the surgeon himself. This study is reassuring concerning the reliability of preoperative planning, but it does not cover the reliability of intra- and post-operative.

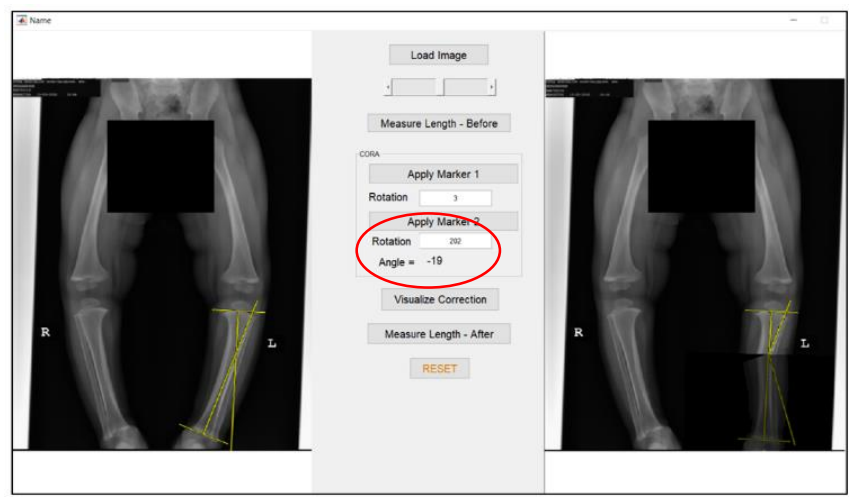

Fig. 4. Finding CORA and Automatically Software will Define Correction Angle.
The outcome was that both current and newly developed software had outstanding reliabilities and consistency in finding CORA for preoperative planning open wedge high tibial osteotomy. In addition, the finding of this paper are comparable to that of Sivertsen [23] in their study comparing the correction angle between Dugdale and Miniaci method found that both Intra- and inter-rater reliability was excellent for the correction angle with 0.992 and 0.991 and 0.988 and 0.987 respectively. So et al [24] ICC for medial proximal tibial angle MPTA is $0.844(0.774-0.892)$.

Schroter et al [25] conducted an ICC comparison between two software, namely PreOPlan and mediCAD with ICC values of $0.993(0.990-0.995)$ and 0.995 (0.992-0.996) respectively on the wedge angle. Elson et al. [19] in turn compared the results obtained on digital planning using the Miniaci method where the ICC values for interrater 0.980 0.986 and intra-rater $0.968-0.985$, respectively.

\section{CONCLUSION}

Preoperative planning for HTO has proven useful in producing the best decision needs to be taken in the surgery. However, manual procedures become obsolete and take time. We have developed a new digital preoperative planning for HTO to assist the developer to assist surgeon in their task. The digital method undertakes the manual procedure hence to reduce the time for decision making and giving the more effective way to provide a solution.

Based on the testing that has been done shows that the existing software also has similar results to the new software. This makes the new software can be adopted to conduct testing for future osteotomy prenatal planning as well as provide added value such as post-surgical expectation display and planning that can be repeated using fully digital. This finding also contributes in medical field especially in the digital preoperative planning of HTO.

The principal finding of this study is that, the new software OsteoAid method showed excellent reliabilities and good consistency in preoperative planning. This proves that all hypotheses have failed to reject the null hypothesis meaning to accept the hypotheses. Consequently, it shows the reliability of each rater for all tested software is zero. Thus, the use of the OsteoAid enables direct measurement of the angle measurement with high reliability.

There were various flaws in this study. The clinical results of patients after surgery were not studied, but that was not the study's major goal. The other limitation was this software is focusing on the HTO only. The future study should expand to other practices in orthopedics. It also should enhance 3D medical images in future.

\section{ACKNOWLEDGMENT}

This research project was conducted in collaboration with Prof Dr Abdul Halim Abdul Rashid from the Department of Orthopaedic and Traumatology, Medical Centre of University Kebangsaan Malaysia. University Grants GP-2019-K007341. 


\section{REFERENCES}

[1] C. Jin, E. K. Song, A. Santoso, P. S. Ingale, I. S. Choi, and J. K. Seon, "Survival and Risk Factor Analysis of Medial Open Wedge High Tibial Osteotomy for Unicompartment Knee Osteoarthritis," Arthrosc. - J. Arthrosc. Relat. Surg., vol. 36, no. 2, pp. 535-543, 2020, doi: 10.1016/j.arthro.2019.08.040.

[2] R. M. A. Raja Izaham, M. R. Abdul Kadir, A. H. Abdul Rashid, M. G. Hossain, and T. Kamarul, "Finite element analysis of Puddu and Tomofix plate fixation for open wedge high tibial osteotomy," Injury, vol. 43, no. 6, pp. 898-902, 2012, doi: 10.1016/j.injury.2011.12.006.

[3] H. Kim, H. Lee, J. Shin, K. Park, S. Min, and H. Kyung, "Preoperative planning using the picture archiving and communication system technique in high tibial osteotomy," vol. 25 , no. 1, pp. 1-6, 2017, doi: 10.1177/2309499016684701.

[4] X. Liu, Z. Chen, Y. Gao, J. Zhang, and Z. Jin, "High Tibial Osteotomy: Review of Techniques and Biomechanics," J. Healthc. Eng., vol. 2019, no. December 2018, 2019, doi: 10.1155/2019/8363128.

[5] F. Gebhard et al., "Reliability of computer-assisted surgery as an intraoperative ruler in navigated high tibial osteotomy," Arch Orthop Trauma Surg, vol. 131, pp. 297-302, 2011, doi: 10.1007/s00402-0101145-9.

[6] B. Zampogna et al., "Assessing Lower Limb Alignment: Comparison of Standard Knee Xray vs Long Leg View,” Iowa Orthop. J., vol. 35, pp. 49-54, 2015, [Online]. Available: http://www.ncbi.nlm.nih.gov/pubmed /26361444\%0Ahttp://www.ncbi.nlm.nih.gov/pmc/articles/PMC4492139 /pdf/IOJ_2015_49.pdf.

[7] Q. L. H. T. T. Nguyen, P. T. Nguyen, V. D. B. Huynh, and L. T. Nguyen, "Application Chang's extent analysis method for ranking barriers in the e-learning model based on multi-stakeholder decision making," Univers. J. Educ. Res., vol. 8, no. 5, pp. 1759-1766, 2020, doi: 10.13189/ujer.2020.080512.

[8] C. A. S. Velando and E. G. C. Gutierrez, "2D/3D registration with rigid alignment of the pelvic bone for assisting in total hip arthroplasty preoperative planning," Int. J. Adv. Comput. Sci. Appl., vol. 11, no. 5, pp. 681-688, 2020, doi: 10.14569/IJACSA.2020.0110583.

[9] M. F. Alrifaie, Z. H. Ahmed, A. S. Hameed, and M. L. Mutar, "Using Machine Learning Technologies to Classify and Predict Heart Disease," Int. J. Adv. Comput. Sci. Appl., vol. 12, no. 3, pp. 123-127, 2021, doi: 10.14569/IJACSA.2021.0120315.

[10] A. Shapi, "Digital Preoperative Planning for Total Hip Replacement using Two Dimensional X-ray Imaging," Int. J. Comput. Appl., vol. 17, no. 2, pp. 20-27, 2011.

[11] N. Awang, R. Sulaiman, A. Shapi, M. F. M. A. Abdul Halim Abdul Rashid, and S. Osman, "A Comparative Study of Computer Aided System Preoperative Planning for High Tibial Osteotomy," Springer Int. Publ. Switz., vol. LNCS 9429, pp. 189-198, 2015, doi: 10.1007/978-3319-25939-0.

[12] D. Paley, Principles of Deformity Correction. Maryland: Springer, 2003.

[13] J. A. Fixsen, R. A. Hill, F. Grill, J. A. Fixsen, and R. A. Hill, Leg Deformity and Length Discrepancy Part I: Classification and Management of Lower Limb Reduction Anomalies. 2010.
[14] P. Gupta, V. Gupta, B. Patil, and V. Verma, "Angular deformities of lower limb in children: Correction for whom, when and how?," J. Clin. Orthop. Trauma, vol. 11, no. 2, pp. 196-201, 2020, doi: 10.1016/j.jcot.2020.01.008.

[15] K. Igarashi et al., "Distal Tibial Tuberosity Focal Dome Osteotomy Combined With Intra-Articular Condylar Osteotomy (Focal Dome Condylar Osteotomy) for Medial Osteoarthritis of the Knee Joint," Arthrosc. Tech., vol. 9, no. 8, pp. e1079-e1086, 2020, doi: 10.1016/j.eats.2020.04.004.

[16] P. Mazdarani, M. B. M. Nielsen, R. S. Gundersen, A. von Wenck, and J. E. Miles, "Geometric modelling of CORA-based levelling osteotomy in the dog," Res. Vet. Sci., vol. 135, no. June 2020, pp. 127-133, 2021, doi: 10.1016/j.rvsc.2021.01.005.

[17] J. E. Kim et al., "Difference of preoperative varus-valgus stress radiograph is effective for the correction accuracy in the preoperative planning during open-wedge high tibial osteotomy," Knee Surgery, Sport. Traumatol. Arthrosc., vol. 29, no. 4, pp. 1035-1044, 2021, doi: 10.1007/s00167-020-06076-4.

[18] J. Kim, H. Kim, and D. Lee, "Leg length change after opening wedge and closing wedge high tibial osteotomy: A meta- analysis," pp. 1-10, 2017.

[19] D. W. Elson, T. G. Petheram, and M. J. Dawson, "High reliability in digital planning of medial opening wedge high tibial osteotomy, using Miniaci's method," Knee Surgery, Sport. Traumatol. Arthrosc., vol. 23, no. 7, pp. 2041-2048, 2015, doi: 10.1007/s00167-014-2920-x.

[20] J. Karlsson et al., A Practical Guide to Research: Design, Execution, and Publication, vol. 27, no. 4. Elsevier Inc., 2011.

[21] J. Blackburn, A. Ansari, A. Porteous, and J. Murray, "Reliability of two techniques and training level of the observer in measuring the correction angle when planning a high tibial osteotomy," Knee, vol. 25, no. 1, pp. 130-134, 2018, doi: 10.1016/j.knee.2017.11.007.

[22] S. Galal, "The Resolution Axis Method (RAM) for lengthening of the femur with or without associated frontal plane deformity (a new method)," Strateg. Trauma Limb Reconstr., vol. 13, no. 2, pp. 109-118, 2018, doi: 10.1007/s11751-018-0312-3.

[23] E. A. Sivertsen, J. Vik, A. S. V. Meland, and T. K. Nerhus, "The Dugdale planning method for high tibial osteotomies underestimates the correction angle compared to the Miniaci method," Knee Surgery, Sport. Traumatol. Arthrosc., 2021, doi: 10.1007/s00167-021-06663-Z.

[24] S. Y. So, S. S. Lee, E. Y. Jung, J. H. Kim, and J. H. Wang, "Difference in joint line convergence angle between the supine and standing positions is the most important predictive factor of coronal correction error after medial opening wedge high tibial osteotomy," Knee Surgery, Sport. Traumatol. Arthrosc., vol. 28, no. 5, pp. 1516-1525, 2020, doi: 10.1007/s00167-019-05555-7.

[25] S. Schroter, C. Ihle, J. Mueller, P. Lobenhoffer, U. Stockle, and R. van Heerwaarden, "Digital planning of high tibial osteotomy . Interrater reliability by using two different software," vol. 21, pp. 189-196, 2013, doi: 10.1007/s00167-012-2114-3. 